Cancer (IARC), Lyon, France, ${ }^{4}$ Department of Epidemiology and Public Health, Swiss Tropical and Public Health Institute, Basel, Switzerland, ${ }^{5}$ University of Basel, Basel, Switzerland, ${ }^{6}$ The Cancer Registry of Norway, Oslo, Norway, ${ }^{7}$ National Institute of Occupational Health, Oslo, Norway, ${ }^{8}$ Department of Oncology, University Children's Hospital of Zurich, Zurich, Switzerland, ${ }^{9}$ Unit of Survivorship, The Danish Cancer Society Research Centre, Copenhagen, Denmark, ${ }^{10}$ Oncology Department, Finsen Centre, Copenhagen, Denmark, ${ }^{11}$ Swiss Childhood Cancer Registry, Institute of Social and Preventive Medicine, University of Bern, Bern, Switzerland, ${ }^{12}$ Childrens Cancer Center, Queen Silvia Childrens Hospital, Gothenburg, Sweden, ${ }^{13}$ Department of Clinical Genetics, University Hospital Rigshospitalet, Copenhagen, Denmark.

BACKGROUND: Knowledge on the role of genetic polymorphisms in the etiology of pediatric brain tumors (PBTs) is limited. Therefore, we investigated the association between single nucleotide polymorphisms (SNPs) identified by candidate gene-association studies on adult brain tumors, and PBT risk. METHODS: The study is based on the largest series of PBT cases to date. Saliva DNA from 245 cases and 489 controls, aged 7-19 years at diagnosis/reference date, was genotyped for 68 SNPs. Data were analyzed using unconditional logistic regression. Stratified analyses were conducted for two histological subtypes: astrocytoma alone and other tumor types combined. RESULTS: EGFR rs730437 (p=0.002) and EGFR rs11506105 $(\mathrm{p}=0.036)$ may decrease susceptibility to PBTs, whereas ERCC1 rs3212986 $(\mathrm{p}=0.009)$ may increase risk of these tumors. Moreover, stratified analyses indicated that CHAF1A rs243341, CHAF1A rs2992, and XRCC1 rs25487 were associated with a decreased risk of astrocytoma subtype $\left(\mathrm{p}_{\mathrm{DOM}}=0.040, \mathrm{p}_{\mathrm{DOM}}=0.049\right.$, and $\mathrm{p}_{\mathrm{DOM}}=0.033$, respectively $)$. In addition, an increased risk of non-astrocytoma tumor subtype associated with EGFR rs9642393, EME1 rs12450550, ATM rs170548, and GLTSCR1 rs1035938 $\left(\mathrm{p}_{\mathrm{REC}}=0.021, \mathrm{p}_{\mathrm{REC}}=0.001, \mathrm{p}_{\mathrm{DOM}}=0.041\right.$, and $\mathrm{p}_{\mathrm{REC}}=0.027$, respectively $)$ as well as a decreased risk of this subtype associated with XRCC4 rs7721416 $\left(\mathrm{p}_{\mathrm{REC}}=0.032\right)$ and XRCC4 $\mathrm{rs} 2662242\left(\mathrm{p}_{\mathrm{REC}}=0.024\right)$ were detected. CON CLUSIONS: This study indicates that SNPS in EGFR, ERCC1, CHAF1A XRCC1, EME1, ATM, GLTSCR1, and XRCC4 may be associated with the risk of PBTs. Therefore, genetic polymorphisms in cell cycle and DNA repair pathways associated with susceptibility to adult brain tumors also seem to be associated with PBT risk, suggesting that pediatric and adult brain tumors might share similar etiological pathways.

\section{GENE-09. PRECISION MEDICINE ANALYSIS OF 203 PEDIATRIC BRAIN TUMORS REVEALS CLINICALLY RELEVANT GENOMIC ALTERATIONS}

Pratiti Bandopadhayay $^{1,2}$, Shakti Ramkissoon ${ }^{3,4}$, Jaeho Hwang ${ }^{3,6}$, Lori Ramkissoon $^{3,4}$, Noah Greenwald ${ }^{2,3}$, Steven Schumacher ${ }^{2,3}$ Ryan O'Rourke ${ }^{2,3}$, Nathan Pinches ${ }^{1}$, Patricia $\mathrm{Ho}^{2,3}$, Hayley Malkin ${ }^{1}$, Claire Sinai ${ }^{1}$, Mariella Filbin ${ }^{1,2}$, Ashley Plant ${ }^{1}$, Wenya $\mathrm{Bi}^{3,4}$

Michael Chang ${ }^{1}$, Edward Yang ${ }^{1}$, Karen Wright ${ }^{1}$, Peter Manley ${ }^{1}$, Matthew Ducar ${ }^{3}$, Sanda Alexandrescu ${ }^{1}$, Hart Lidov ${ }^{5}$, Ivana Delalle ${ }^{4}$, Liliana Goumnerova ${ }^{1,4}$, Alanna Church ${ }^{5}$, Katherine Janeway ${ }^{1}$, Marian Harris ${ }^{3,4}$, Laura MacConaill ${ }^{4}$, Rebecca Folkerth ${ }^{4,5}$, Neal Lindeman ${ }^{3,4}$, Charles Stiles ${ }^{3}$, Mark Kieran ${ }^{1}$, Azra Ligon ${ }^{4}$, Sandro Santagata ${ }^{4,5}$, Adrian Dubuc ${ }^{4}$, Susan Chi ${ }^{* 1}$, Rameen Beroukhim ${ }^{* 2,3}$, and Keith Ligon ${ }^{3,4}$; ${ }^{1}$ Dana-Farber/Boston Children's Cancer and Blood Disorders Center, Boston, MA, USA, ${ }^{2}$ Broad Institute of MIT and Harvard, Boston, MA, USA, ${ }^{3}$ Dana-Farber Cancer Institute, Boston, MA, USA, ${ }^{4}$ Brigham and Women's Hospital, Boston, MA, USA, ${ }^{5}$ Boston Children's Hospital, Boston, MA, USA, ${ }^{6}$ Harvard Medical School, Boston, MA, USA

INTRODUCTION: Clinical genomics platforms identify targetable alterations for precision medicine in pediatric neuro-oncology, allowing both the genomic profiling of tumors to inform clinical care and facilitating the potential discovery of novel driver alterations. METHODS: We applied two clinical genomic platforms (OncoPanel and OncoCopy) to profile pediatric brain tumors in a clinical setting. OncoPanel, a multiplexed targeted exomesequencing platform that includes 300 cancer causing genes, was used to assess single nucleotide variants and rearrangements/indels. OncoCopy, a high-resolution genome-wide array comparative genomic hybridization $(\mathrm{aCGH})$ assay, was used to evaluate copy number alterations and refine rearrangement breakpoints. RESULTS: Cancer genomes of 203 pediatric brain tumors were profiled across histological subtypes including 117 analyzed by OncoPanel, 146 by OncoCopy, and 60 evaluated using both methodologies. OncoPanel revealed clinically relevant alterations in 56\% of patients (44 cancer mutations and 20 rearrangements), including BRAF alterations that directed the use of targeted inhibitors. EWSRa rearrangements were observed unexpectedly in two patients; one with a Atypical Teratoid Rhabdoid Tumor with loss of INI-1 (SMARCB1), and the second in an ependymoma. Whole Genome Sequencing of the rhabdoid tumor confirmed a rearrangement involving EWSR1 and PLAGL1. CONCLUSIONS: The combined deployment of OncoPanel and OncoCopy multiplex genomic assays can identify clinically relevant genomic alterations in pediatric brain tumors and facilitate the discovery of novel driver alterations. "equal contribution

GENE-10. H3K27ME3-MEDIATED SILENCING TUMOR SUPPRESSORS SUPPORTS THE PROLIFERATION OF PEDIATRIC BRAIN TUMOR CELLS HARBORING THE H3.3K27M MUTATION Dong Fang ${ }^{1}$, Haiyun Gan ${ }^{1}$, Liang Cheng ${ }^{2}$, Jeong-Heon Lee ${ }^{2}$, Hui Zhou ${ }^{1}$, Jann Sarkaria ${ }^{2}$, David Daniels ${ }^{2}$, and Zhiguo Zhang ${ }^{1}$; ${ }^{1}$ Columbia University Medical Center, New York, NY, USA, ${ }^{2}$ Mayo Clinic, Rochester, MN, USA.

Recent genetic studies have shown that the lysine 27 to methionine of histone H3.3 (H3.3K27M) mutant proteins expressed in over $75 \%$ of diffuse intrinsic pontine glioma (DIPG) cases inhibit H3K27 methyltransferase PRC2, resulting in a global loss of tri-methylation of H3K27 (H3K27me3). In addition, H3K27me3 is also retained at hundreds of gene loci. However it is not known how and why H3K27me3 is retained in DIPG cells. Here we show that $\mathrm{H} 3 \mathrm{~K} 27 \mathrm{me} 3$ is enriched on chromatic loci where the levels of H3.3K27M mutant proteins are low. Furthermore, H3K27me3-mediated silencing of tumor suppressor genes supports the proliferation of DIPG cells. Together, these studies reveal mechanisms whereby $\mathrm{H} 3 \mathrm{~K} 27 \mathrm{me} 3$ is retained in the environmental of global loss of this mark and the functional significance of retention of this mark in tumorigenesis.

\section{GENE-11. LSD1/KDM1A MODULATES AN ONCO-IMMUNOGENIC} GENE SIGNATURE IN ADULT GBM AND EPIGENOME-MUTATED PEDIATRIC DIPG

Cavan Bailey ${ }^{1,2}$, Melissa Singh ${ }^{1}$, and Joya Chandra ${ }^{1,2} ;{ }^{1}$ Department of Pediatrics - Research, MD Anderson Cancer Center, Houston, TX, USA, ${ }^{2}$ The University of Texas MD Anderson Cancer Center UTHealth Graduate School of Biomedical Sciences, Houston, TX, USA, ${ }^{3}$ Center for Cancer Epigenetics, MD Anderson Cancer Center, Houston, TX, USA.

Diffuse intrinsic pontine glioma (DIPG) patients often possess a lysineto-methionine mutation in histone $\mathrm{H} 3(\mathrm{H} 3.1 / .3-\mathrm{K} 27 \mathrm{M})$ that renders them sensitive to perturbations in histone methylation. However, the demethylase KDM1A/LSD1 has not yet been investigated as a therapeutic target in DIPG. Our lab has studied LSD1 in adult GBM, generating RNA-Seq data from LSD1 knockdown cells, for which DAVID pathway analysis was performed with a 1.5 -fold change filter. Significantly changed was a set of immune response genes (18 upregulated, 6 downregulated) including stimulatory ligands, antigen presentation and cell motility genes. To validate, LSD1 was knocked down via shRNA in LN18 cells and RT-qPCR performed using unique primers for 13 of the most upregulated immunomodulatory genes. T-tests were significant $(\mathrm{p}<0.05)$ for $5 / 13$ of these genes, and one-way ANOVA analysis of all genes was highly significant $(\mathrm{p}<0.0001)$ versus scramble control. We further hypothesized that LSD1 chemical inhibitors (LSD1i) could replicate these immunomodulatory gene changes in LN18 cells, as well as in DIPG cells bearing K27M mutations. SLAMF7, 4-1BB, and LCP1 were most significantly changed in LN18 cells across LSD1i treatments via T-test $(\mathrm{p}<0.05)$. DIPG cells showed significant data via T-test $(\mathrm{p}<0.05)$ for SLAMF7, RAET1E, and MICB. Importantly, ANOVA comparison of data between TCP-treated LN18 and DIPG cells, as well as LN18 LSD1i and LSD1-KD, was not significant ( $q>0.9999)$, indicating their induced immunomodulatory gene expression profiles are similar. Interestingly, cytotoxicity of tranylcypromine (TCP), GSK LSD1, and RN-1 was tested, with LN18 significantly more resistant to LSD1i cytotoxicity than DIPG cells, indicating LSD1 may play a key role in DIPG survival. TCP is FDA-approved and the only LSD1 inhibitor known to pass through the blood-brain barrier, which is critical for its use as a potential glioma therapeutic. Ongoing studies are examining if LSD1i can enhance immunotherapeutic efficacy against target cells via our documented gene changes.

GENE-12. THE CHILDREN'S BRAIN TUMOR TISSUE CONSORTIUM (CBTTC) INFRASTRUCTURE FACILITATES COLLABORATIVE RESEARCH IN PEDIATRIC CENTRAL NERVOUS SYSTEM TUMORS

Alex Felmeister ${ }^{1,5}$, Rishi Lulla ${ }^{3,4}$, Angela Waanders ${ }^{1,2}$, Pichai Raman ${ }^{1}$, Mariarita Santi ${ }^{1,2}$, Jena Lilly ${ }^{1}$, Jennifer Mason ${ }^{1}$, Javad Nazarian ${ }^{6,7}$, and Adam Resnick ${ }^{1,2}$; ${ }^{1}$ The Children's Hospital of Philadelphia, Philadelphia, PA, USA, ${ }^{2}$ Perelman School of Medicine At the University of Pennsylvania, Philadelphia, PA, USA, ${ }^{3}$ Ann \& Robert H. Lurie Children's Hospital of Chicago, Chicago, IL, USA, ${ }^{4}$ Northwestern University Feinberg School of Medicine, Chicago, IL, USA, ${ }^{5}$ Drexel University, Philadelphia, PA, USA, ${ }^{6}$ Children's National Medical Center, Washington, DC, USA, ${ }^{7}$ George Washington University School of Medicine and Health Sciences, Washington, DC, USA

INTRODUCTION: The Children's Brain Tumor Tissue Consortium (CBTTC) is a multi-institutional, international research collaboration comprised of 13 institutions utilizing an infrastructure of web based open source tools to accelerate pediatric brain tumor research. The CBTTC mission is 